\title{
Dependence of polymer thin film adhesion energy on cohesive interactions between chains
}

\author{
Xia, Wenjie, wenjiexia2011@u.northwestern.edu; Hsu, David; Keten, Sinan, Northwestern University,
} United States

\begin{abstract}
The adhesion of supported polymer thin films is predominantly influenced by the substrate-film interfacial properties. Utilizing steered molecular dynamics simulations, here we uncover that the cohesive noncovalent forces between polymer chains in the film also have a significant effect on the adhesive properties of supported film. We demonstrate that weaker interchain interactions, all else being the same, can induce higher adhesion energy within the interface. Three different adhesion regimes in the substrate-film interaction strength profile can be characterized by a nonlinear scaling relationship that earlier theoretical predictions currently do not capture. In the weak substrate-film interaction regime, the adhesion energy of the films exhibits near independence of cohesive forces, and entropic contributions to the surface free energy are consequential. In the intermediate regime, weaker film cohesive forces achieve higher adhesion energy due to the ability of polymer chains to pack more effectively in the interfacial region, thereby increasing the adhesive interaction density. In the strong interaction regime, the adhesion energy increases linearly with the adhesive interaction strength because of saturation of local packing in the interfacial region. These findings corroborate recent polymer wetting observations that have hinted on the importance of local relaxation and packing effects on interfacial properties.
\end{abstract}

